TRANSACTIONS OF THE

AMERICAN MATHEMATICAL SOCIETY

Volume 363, Number 1, January 2011, Pages 501-519

S 0002-9947(2010)05149-9

Article electronically published on August 30, 2010

\title{
THIN-VERY TALL COMPACT SCATTERED SPACES WHICH ARE HEREDITARILY SEPARABLE
}

\author{
CHRISTINA BRECH AND PIOTR KOSZMIDER
}

\begin{abstract}
We strengthen the property $\Delta$ of a function $f:\left[\omega_{2}\right]^{2} \rightarrow\left[\omega_{2}\right] \leq \omega$ considered by Baumgartner and Shelah. This allows us to consider new types of amalgamations in the forcing used by Rabus, Juhász and Soukup to construct thin-very tall compact scattered spaces. We consistently obtain spaces $K$ as above where $K^{n}$ is hereditarily separable for each $n \in \mathbb{N}$. This serves as a counterexample concerning cardinal functions on compact spaces as well as having some applications in Banach spaces: the Banach space $C(K)$ is an Asplund space of density $\aleph_{2}$ which has no Fréchet smooth renorming, nor an uncountable biorthogonal system.
\end{abstract}

\section{INTRODUCTION}

Given a compact scattered space $K$, we call the derivative of $K$ (denoted by $K^{\prime}$ ) the subset of $K$ formed by its accumulation points, and we inductively define $K^{(\alpha)}=\left(K^{(\beta)}\right)^{\prime}$ if $\alpha=\beta+1$ and $K^{(\alpha)}=\bigcap_{\beta<\alpha} K^{(\beta)}$ if $\alpha$ is a limit ordinal. The height of $K, h t(K)$, is the smallest ordinal $\alpha$ such that $K^{(\alpha)}$ is finite and nonempty, and the width of $K, w d(K)$, is the supremum of the cardinalities $\left|K^{(\alpha)} \backslash K^{(\alpha+1)}\right|$ for $\alpha<$ $h t(K)$. We call $K=\bigcup_{\alpha<h t(K)} K^{(\alpha)} \backslash K^{(\alpha+1)}$ the Cantor-Bendixson decomposition of $K$ and $K^{(\alpha)} \backslash K^{(\alpha+1)}$ its $\alpha^{\text {th }}$ Cantor-Bendixson level.

The purpose of this work is to show that the existence of compact hereditarily separable scattered spaces of height $\omega_{2}$ is consistent with the usual axioms of set theory. For a given ordinal $\theta$ let us consider the following notation:

- A $\operatorname{cw}(\theta)$ space is a compact scattered space of countable width and height equal to $\theta$.

- An hs $(\theta)$ space is a compact scattered space which is hereditarily separable and of height equal to $\theta$.

$\operatorname{cw}\left(\omega_{1}\right)$ spaces are usually called thin-tall spaces and $\mathrm{cw}\left(\omega_{2}\right)$ spaces are the thin-very tall spaces. First we remark that any $\mathrm{hs}(\theta)$ space is a $\operatorname{cw}(\theta)$ space as the CantorBendixson levels form discrete subspaces. Whether there is or is not in ZFC a

Received by the editors August 26, 2008 and, in revised form, June 24, 2009.

2010 Mathematics Subject Classification. Primary 54G12; Secondary 03E35, 46B26.

The research was part of Thematic Project FAPESP (2006/02378-7). The first author was supported by scholarships from CAPES (3804/05-4) and CNPq (140426/2004-3 and 202532/20062 ). She would like to thank Stevo Todorcevic and the second author, her Ph.D. advisors at the University of São Paulo and at the University of Paris 7, under whose supervision the results of this paper were obtained.

The second author was partially supported by Polish Ministry of Science and Higher Education research grant N N201 386234. 
cw $\left(\omega_{1}\right)$ space was a question posed by Telgársky in 1968 (unpublished) and first (consistently) answered by Ostaszewski [21, using $\diamond$. Rajagopalan constructed the first ZFC example of a $\mathrm{cw}\left(\omega_{1}\right)$ in 23. Further, Juhász and Weiss generalized these results (and simplified their proofs) in 12 proving in ZFC that for any ordinal $\theta<\omega_{2}$, there is a $\mathrm{cw}(\theta)$ space.

For higher $\theta$ 's the situation changes: in any model of $\mathrm{CH}$ there are no $\mathrm{cw}\left(\omega_{2}\right)$ spaces and Just proved in 13 that neither are there such spaces in the Cohen model (where $\neg \mathrm{CH}$ holds). On the other hand, Baumgartner and Shelah 2 constructed by forcing the first consistent example of a $\mathrm{cw}\left(\omega_{2}\right)$ space. An interesting point of this forcing construction was the use of a new combinatorial device, called a function with the property $\Delta$.

The main purpose of this work is to prove the consistency of the existence of an hs $\left(\omega_{2}\right)$ space. In fact, our space has even stronger properties: each of its finite powers is hereditarily separable. Whether consistently there are $h s\left(\omega_{3}\right)$ or even $\operatorname{cw}\left(\omega_{3}\right)$ spaces remains a well-known open question. On the other hand, Martínez in [17] adopted the method of 2] to obtain the consistency of the existence of $\mathrm{cw}(\theta)$ spaces for each $\theta<\omega_{3}$.

It follows from an old result of Shapirovskiu 25 that for any compact space $K$, $h d(K) \leq h L(K)^{+}$. Our construction shows that the dual inequality does not follow from ZFC, since for our compact space $K$, we have that $h L(K)=\aleph_{2} \not \leq \aleph_{1}=$ $h d(K)^{+}$. Nevertheless, the dual inequality holds under GCH for regular spaces: since the weight $w(K)$ of a regular space $K$ is less than or equal to $2^{d(K)}$ (see, for example, [8]), we trivially conclude that $h L(K) \leq w(K) \leq 2^{d(K)}=d(K)^{+} \leq$ $h d(K)^{+}$under GCH.

Turning to properties of Banach spaces, let us first recall some definitions and results: a Banach space $X$ is an Asplund space if every continuous and convex real-valued function on $X$ is Fréchet smooth at all points of a $\mathrm{G}_{\delta}$ dense subset of $X$. For separable Banach spaces, this is equivalent to admitting a Fréchet smooth renorming (see [4). Namioka and Phelps proved in [19] that $C(K)$ is Asplund if and only if $K$ is scattered. Thus, our $C(K)$ is an Asplund space.

Haydon constructed in $[7$ the first nonseparable Asplund space $C(K)$ which does not admit a Fréchet smooth renorming, concluding that the situation changes for nonseparable Asplund spaces. Later, Jiménez Sevilla and Moreno analyzed in 10. the structural properties of the space $C(K)$, where $K$ is the well-known Kunen line constructed under $\mathrm{CH}$ (see [20]). They showed, for the Kunen line $K$, that $C(K)$ is also a nonseparable Asplund space with no Fréchet smooth renorming.

The weight of our space $K$ is $\aleph_{2}$, so that $C(K)$ is an Asplund space of density $\aleph_{2}$. The fact that $K$ is compact scattered and every finite power of $K$ is hereditarily separable implies, in the same way as for the Kunen line, that $C(K)$ does not admit any Fréchet smooth renorming, but as in the case of the Kunen line we do not know if it admits a Gâteaux smooth renorming, or a Fréchet smooth bump function.

A biorthogonal system on a Banach space $X$ is a family $\left(x_{\alpha}, \varphi_{\alpha}\right)_{\alpha<\kappa} \subseteq X \times X^{*}$ such that $\varphi_{\alpha}\left(x_{\beta}\right)=\delta_{\alpha, \beta}$, and a semi-biorthogonal system on a Banach space $X$ is a sequence $\left(x_{\alpha}, \varphi_{\alpha}\right)_{\alpha<\kappa} \subseteq X \times X^{*}$ such that $\varphi_{\alpha}\left(x_{\beta}\right)=1$ if $\alpha=\beta, \varphi_{\alpha}\left(x_{\beta}\right)=0$ if $\alpha<\beta$ and $\varphi_{\alpha}\left(x_{\beta}\right) \geq 0$ if $\beta<\alpha$. Todorcevic showed in 29] (Theorem 9 together with the results of [3]) the existence of uncountable semi-biorthogonal systems in Banach spaces $C(K)$ of density strictly greater than $\aleph_{1}$. On the other hand, the fact that our space $K$ is compact scattered and every finite power of $K$ is hereditarily 
separable implies, in the same way as for the Kunen line, that $C(K)$ does not admit an uncountable biorthogonal system. It follows that Todorcevic's result cannot be improved in ZFC by replacing the existence of uncountable semi-biorthogonal systems by the existence of uncountable biorthogonal systems in spaces $C(K)$ of large density. On the other hand it is proved in 29] that it is consistent that every nonseparable Banach space has an uncountable biorthogonal system, showing that the existence of a Banach space such as ours or Kunen's cannot be proved in ZFC.

Our construction is based on the Juhász and Soukup [11] interpretation of Rabus' work 22, where he modified the Baumgartner-Shelah forcing from [2] to obtain a countably tight space which is initially $\omega_{1}$-compact and noncompact, answering a question of Dow and van Douwen.

This paper is organized as follows: we finish this section by reviewing the method of Juhász and Soukup and some related results and definitions which we will need afterwards. In Section 2 we prove the key lemma which enables us to prove our main result in a straightforward way. This lemma introduces a new way of amalgamating conditions in forcings which add thin-very tall spaces. One can apply these amalgamations in the generic construction if one strengthens the property $\Delta$ of a function involved in the forcing. In Section 3, we introduce the strong property $\Delta$ and, assuming the existence of a function which satisfies it, we prove the main results and analyze their consequences in topological and functional analytic terms. Section 4 is devoted to establishing the consistency of the existence of a function with the strong property $\Delta$. Section 4 is due to the second author and the remaining sections to the first author.

The notation and terminology used are those of [11. Given a set $X, \wp(X)$ is the power set of $X$ and, given a cardinal $\kappa,[X]^{\kappa}$ (resp. $[X]^{\leq \kappa}$ and $[X]^{<\kappa}$ ) denotes the family of subsets of $X$ of cardinality equal to $\kappa$ (resp. less than or equal to $\kappa$ and less than $\kappa$ ).

Let us start by recalling the definition of the property $\Delta$ :

Definition 1.1 (Baumgartner, Shelah, [2, p.122). A function $f:\left[\omega_{2}\right]^{2} \rightarrow\left[\omega_{2}\right]^{\leq \omega}$ has the property $\Delta$ if $f(\{\xi, \eta\}) \subseteq \min \{\xi, \eta\}$ for all $\{\xi, \eta\} \in\left[\omega_{2}\right]^{2}$ and for any uncountable family $\mathcal{A}$ of finite subsets of $\omega_{2}$, there are distinct $a, b \in \mathcal{A}$ such that for any $\tau \in a \cap b$, any $\xi \in a \backslash b$ and any $\eta \in b \backslash a$ we have:

1) $a \cap b \cap \min \{\xi, \eta\} \subseteq f(\{\xi, \eta\})$;

2) $\tau<\xi \Rightarrow f(\{\tau, \eta\}) \subseteq f(\{\xi, \eta\})$;

3) $\tau<\eta \Rightarrow f(\{\tau, \xi\}) \subseteq f(\{\xi, \eta\})$.

Now, we fix a function $f:\left[\omega_{2}\right]^{2} \rightarrow\left[\omega_{2}\right]^{\leq \omega}$ with the property $\Delta$.

Definition 1.2 (Juhász, Soukup [11, Definition 2.1). Let $\mathbb{P}_{f}$ be the forcing formed by conditions $p=\left(D_{p}, h_{p}, i_{p}\right)$, where:

1. $D_{p} \in\left[\omega_{2}\right]^{<\omega}$,

2. $h_{p}: D_{p} \rightarrow \wp\left(D_{p}\right)$ and for all $\xi \in D_{p}, \max h_{p}(\xi)=\xi$,

3. $i_{p}:\left[D_{p}\right]^{2} \rightarrow\left[D_{p}\right]<\omega$ and for all $\xi, \eta \in D_{p}, \xi<\eta$, we have that:

(a) if $\xi \in h_{p}(\eta)$, then $h_{p}(\xi) \backslash h_{p}(\eta) \subseteq \bigcup_{\gamma \in i_{p}(\{\xi, \eta\})} h_{p}(\gamma)$,

(b) if $\xi \notin h_{p}(\eta)$, then $h_{p}(\xi) \cap h_{p}(\eta) \subseteq \bigcup_{\gamma \in i_{p}(\{\xi, \eta\})} h_{p}(\gamma)$,

(c) $i_{p}(\{\xi, \eta\}) \subseteq f(\{\xi, \eta\})$,

ordered by $p \leq q$ if $D_{p} \supseteq D_{q}$, for all $\xi \in D_{q}, h_{p}(\xi) \cap D_{q}=h_{q}(\xi)$ and $\left.i_{p}\right|_{\left[D_{q}\right]^{2}}=i_{q}$. 
To simplify notation, it is convenient to define the following:

Definition 1.3 (Juhász, Soukup [11]). Given finite nonempty sets of ordinals $x$ and $y$ such that $\max x<\max y$, we define

$$
x * y= \begin{cases}x \backslash y & \text { if } \max x \in y, \\ x \cap y & \text { if } \max x \notin y .\end{cases}
$$

We now rewrite conditions $3(\mathrm{a})$ and $3(\mathrm{~b})$ of the definition of the forcing as

$$
h_{p}(\xi) * h_{p}(\eta) \subseteq \bigcup_{\gamma \in i_{p}(\{\xi, \eta\})} h_{p}(\gamma) .
$$

To define the space $K_{f}$, fix the ground model $V$ and a generic filter $G$.

Definition 1.4 (Juhász, Soukup [1], Definition 2.3). For each $\xi<\eta<\omega_{2}$, working in $V^{\mathbb{P}_{f}}$, let

$$
h(\xi)=\bigcup_{p \in G} h_{p}(\xi) \quad \text { and } \quad i(\{\xi, \eta\})=\bigcup_{p \in G} i_{p}(\{\xi, \eta\}),
$$

and let $L_{f}$ be the topological space $\left(\omega_{2}, \tau\right)$, where $\tau$ is the topology on $\omega_{2}$ which has the family of sets

$$
\left\{h(\xi): \xi<\omega_{2}\right\} \cup\left\{\omega_{2} \backslash h(\xi): \xi<\omega_{2}\right\}
$$

as a topological subbasis. We call $h(\xi)$ the generic neighborhood of $\xi$.

From Theorem 1.5 of [11, it follows that for all $\xi<\omega_{2}, h(\xi)$ is a compact subspace of $\left(\omega_{2}, \tau\right)$ and it easy to check that

(+) $\quad\left\{h(\xi) \backslash \bigcup_{\eta \in F} h(\eta): F \in[\xi]^{<\omega}\right\}$ forms a local topological basis at $\xi$.

Therefore $L_{f}$ is a locally compact scattered zero-dimensional space.

We are now ready to define $K_{f}$ :

Definition 1.5. In $V^{\mathbb{P}_{f}}, K_{f}$ is the one-point compactification of $L_{f}$. The point of compactification is denoted $*$; thus $K_{f} \backslash L_{f}=\{*\}$.

In particular, we use the following results.

Theorem 1.6 (Rabus [22, Lemma 4.1; Juhász, Soukup [1], Lemma 2.8). $\mathbb{P}_{f}$ satisfies c.c.c.

Proposition 1.7. $V^{\mathbb{P}_{f}}$ satisfies " $K_{f}$ is a compact scattered zero-dimensional space".

\section{Amalgamating Conditions}

In this section, we present the key lemma needed to prove our main result. Let us start with some preliminaries and auxiliary lemmas.

Definition 2.1. Let $p_{1}=\left(D_{1}, h_{1}, i_{1}\right), p_{2}=\left(D_{2}, h_{2}, i_{2}\right) \in \mathbb{P}_{f}$ be two conditions. We say that $p_{1}$ and $p_{2}$ are isomorphic conditions if there is an order-preserving bijective function $e: D_{1} \rightarrow D_{2}$ satisfying the following conditions:

(a) if $\xi, \eta \in D_{1}$, then $\xi \in h_{1}(\eta)$ if and only if $e(\xi) \in h_{2}(e(\eta))$;

(b) if $\xi \in D_{1} \cap D_{2}$, then $e(\xi)=\xi$.

In this case, if the order-preserving bijection $e$ is such that $\xi \leq e(\xi)$ for every $\xi \in D_{1}$ we say that $p_{1}$ is lower than $p_{2}$. 
For example we have the following:

Lemma 2.2. Let $p_{1}=\left(D_{1}, h_{1}, i_{1}\right), p_{2}=\left(D_{2}, h_{2}, i_{2}\right) \in \mathbb{P}_{f}$ be two isomorphic conditions and let $e: D_{1} \rightarrow D_{2}$ be the order-preserving bijection. Then for every $\xi \in D_{1} \cap D_{2}$,

(a) $\left(h_{1}(\xi) \cup h_{2}(\xi)\right) \cap D_{1}=h_{1}(\xi)$,

(b) $\left(h_{1}(\xi) \cup h_{2}(\xi)\right) \cap D_{2}=h_{2}(\xi)$,

(c) $h_{1}(\xi)=e^{-1}\left[h_{2}(\xi)\right]$.

Proof. Directly from Definition 2.1.

Definition 2.3 (Juhász, Soukup [1]). Given $p_{1}=\left(D_{1}, h_{1}, i_{1}\right), p_{2}=\left(D_{2}, h_{2}, i_{2}\right) \in$ $\mathbb{P}_{f}$, define a mapping $\delta_{2}: \operatorname{dom}\left(\delta_{2}\right) \rightarrow D_{1} \cap D_{2}$, where

$$
\operatorname{dom}\left(\delta_{2}\right)=\left\{\eta \in D_{2}: \text { there is } \delta \in D_{1} \cap D_{2} \text { such that } \eta \in h_{2}(\delta)\right\}
$$

and

$$
\delta_{2}(\eta)=\min \left\{\delta \in D_{1} \cap D_{2}: \eta \in h_{2}(\delta)\right\} .
$$

Lemma 2.4. Suppose that $p_{1}=\left(D_{1}, h_{1}, i_{1}\right)$ and $p_{2}=\left(D_{2}, h_{2}, i_{2}\right) \in \mathbb{P}_{f}$ are two conditions. Then,

(a) for all $\eta \in \operatorname{dom}\left(\delta_{2}\right) \backslash D_{1}$, we have that $\eta<\delta_{2}(\eta)$ and

(b) for all $\eta \in D_{1} \cap D_{2}$ we have $\eta \in \operatorname{dom}\left(\delta_{2}\right)$ and $\delta_{2}(\eta)=\eta$.

Proof. Directly from Definition 2.3.

We prove the next lemma, for the reader's convenience.

Lemma 2.5 (Juhász, Soukup [1] ). Let $p_{1}=\left(D_{1}, h_{1}, i_{1}\right), p_{2}=\left(D_{2}, h_{2}, i_{2}\right) \in \mathbb{P}_{f}$ be two isomorphic conditions. If $\xi \in D_{1} \cap D_{2}$, then

$$
h_{2}(\xi)=\delta_{2}^{-1}\left[h_{1}(\xi)\right]
$$

Proof. Let $\xi \in D_{1} \cap D_{2}$.

Suppose that $\eta \in \operatorname{dom}\left(\delta_{2}\right)$ and $\delta_{2}(\eta) \in h_{1}(\xi)$. Since $\delta_{2}(\eta), \xi \in D_{1} \cap D_{2}$, it follows from Definition 2.1 (a) and (b) that $\delta_{2}(\eta) \in h_{2}(\xi)$. Suppose that $\eta \notin h_{2}(\xi)$. Then, $\eta \in h_{2}\left(\delta_{2}(\eta)\right) * h_{2}(\xi)$ so that there is $\delta \in i_{2}\left(\left\{\delta_{2}(\eta), \xi\right\}\right)$ such that $\eta \in h_{2}(\delta)$, which contradicts the minimality of $\delta_{2}(\eta)$ and concludes the proof of the inclusion $\delta_{2}^{-1}\left[h_{1}(\xi)\right] \subseteq h_{2}(\xi)$.

Reciprocally, if $\eta \in h_{2}(\xi)$, then $\eta \in \operatorname{dom}\left(\delta_{2}\right)$. Suppose that $\delta_{2}(\eta) \notin h_{2}(\xi)$. Then, $\eta \in h_{2}\left(\delta_{2}(\eta)\right) * h_{2}(\xi)$ so that there is $\delta \in i_{2}\left(\left\{\delta_{2}(\eta), \xi\right\}\right)$ such that $\eta \in h_{2}(\delta)$, which contradicts the minimality of $\delta_{2}(\eta)$. So, $\delta_{2}(\eta) \in h_{2}(\xi)$ and since $\delta_{2}(\eta), \xi \in D_{1} \cap D_{2}$, it follows from Definition 2.1 (a) and (b) that $\delta_{2}(\eta) \in h_{1}(\xi)$, concluding the proof of the lemma.

In the proof of c.c.c., Rabus, Juhász and Soukup considered the minimal amalgamation which is constructed in a symmetric way with respect to both of the conditions being extended. We will consider an asymmetric amalgamation. The lack of symmetry in our amalgamation is the result of using two functions, $\delta_{2}$ and $e$, in the definition of the amalgamation. The final auxiliary lemma below characterizes the sets given by the operation $*$ for elements of the extended condition. The role of the function $g$ will be played by $\delta_{2}$ or by $e$. 
Lemma 2.6. Let $p=\left(D_{p}, h_{p}, i_{p}\right) \in \mathbb{P}_{f}$ and let $D_{q} \in\left[\omega_{2}\right]^{<\omega}, h_{q}: D_{q} \rightarrow \wp\left(D_{q}\right)$ and $g: \operatorname{dom}(g) \rightarrow D_{p}$ be such that

(i) $D_{p} \subseteq D_{q}, \operatorname{dom}(g) \subseteq D_{q}$,

(ii) for all $\xi \in D_{p} \cap \operatorname{dom}(g)$ we have $g(\xi)=\xi$,

(iii) for all $\xi \in D_{p}, h_{q}(\xi)=h_{p}(\xi) \cup g^{-1}\left[h_{p}(\xi)\right]$.

Then, for all $\xi, \eta \in D_{p}, \xi<\eta$, we have that

$$
\left(h_{q}(\xi) * h_{q}(\eta)\right) \cap D_{p}=h_{p}(\xi) * h_{p}(\eta)
$$

and

$$
\left(h_{q}(\xi) * h_{q}(\eta)\right) \cap\left(D_{q} \backslash D_{p}\right)=g^{-1}\left[h_{p}(\xi) * h_{p}(\eta)\right] \cap\left(D_{q} \backslash D_{p}\right) .
$$

Proof. Since $\xi, \eta \in D_{p}, \xi<\eta$, by (ii) we have that $\xi \in h_{p}(\eta)$ if and only if $\xi \in h_{q}(\eta)$, so (ii) obviously gives $\left(h_{q}(\xi) * h_{q}(\eta)\right) \cap D_{p}=h_{p}(\xi) * h_{p}(\eta)$.

Now suppose $\xi \in h_{q}(\eta)$, so $\xi \in h_{p}(\eta)$ and so by (iii),

$$
\left(h_{q}(\xi) \backslash h_{q}(\eta)\right) \cap\left(D_{q} \backslash D_{p}\right)=\left(h_{q}(\xi) \cap\left(D_{q} \backslash D_{p}\right)\right) \backslash\left(h_{q}(\eta) \cap\left(D_{q} \backslash D_{p}\right)\right)
$$

$=\left(g^{-1}\left[h_{p}(\xi)\right] \cap\left(D_{q} \backslash D_{p}\right)\right) \backslash\left(g^{-1}\left[h_{p}(\eta)\right] \cap\left(D_{q} \backslash D_{p}\right)\right)=g^{-1}\left[h_{p}(\xi) \backslash h_{p}(\eta)\right] \cap\left(D_{q} \backslash D_{p}\right)$.

On the other hand, if $\xi \notin h_{q}(\eta)$, then $\xi \notin h_{p}(\eta)$ and so by (iii),

$$
\left(h_{q}(\xi) \cap h_{q}(\eta)\right) \cap\left(D_{q} \backslash D_{p}\right)=\left(h_{q}(\xi) \cap\left(D_{q} \backslash D_{p}\right)\right) \cap\left(h_{q}(\eta) \cap\left(D_{q} \backslash D_{p}\right)\right)
$$

$=\left(g^{-1}\left[h_{p}(\xi)\right] \cap\left(D_{q} \backslash D_{p}\right)\right) \cap\left(g^{-1}\left[h_{p}(\eta)\right] \cap\left(D_{q} \backslash D_{p}\right)\right)=g^{-1}\left[h_{p}(\xi) \cap h_{p}(\eta)\right] \cap\left(D_{q} \backslash D_{p}\right)$, concluding the proof of the lemma.

Now we go to our key lemma: a strong hypothesis about the behaviour of the function $f$ allows us to amalgamate two isomorphic conditions, one lower than the other, into a common extension $q$ in such a way that $h(\xi) \cap D_{q} \subseteq h[e(\xi)] \cap D_{q}$ for $\xi$ in the domain of the lower of the two conditions.

Lemma 2.7. Let $p_{1}=\left(D_{1}, h_{1}, i_{1}\right), p_{2}=\left(D_{2}, h_{2}, i_{2}\right) \in \mathbb{P}_{f}$ be two isomorphic conditions and suppose $p_{1}$ is lower than $p_{2}$. Let e : $D_{1} \rightarrow D_{2}$ be the order-preserving bijective function and assume that

(A) if $\xi, \eta \in D_{1} \cap D_{2}$ and $\xi \neq \eta$, then $i_{1}(\{\xi, \eta\})=i_{2}(\{\xi, \eta\})$;

(B) for all $\zeta \in D_{1} \cap D_{2}$, all $\xi \in D_{1} \backslash D_{2}$ and all $\eta \in D_{2} \backslash D_{1}$ :

(i) if $\zeta<\xi$, then $f(\{\zeta, \eta\}) \subseteq f(\{\xi, \eta\})$;

(ii) $D_{1} \cap \xi \cap \eta \subseteq f(\{\xi, \eta\})$.

Then there is $q \in \mathbb{P}_{f}, q \leq p_{1}, p_{2}$, such that for all $\xi \in D_{1}$ and all $\eta \in D_{2}$ :

$$
\xi \in h_{q}(\eta) \text { if and only if } e(\xi) \in h_{2}(\eta) \text {. }
$$

Proof. We define $q=\left(D_{q}, h_{q}, i_{q}\right)$ by: $D_{q}=D_{1} \cup D_{2}$;

$$
h_{q}(\xi)= \begin{cases}h_{1}(\xi) \cup \delta_{2}^{-1}\left[h_{1}(\xi)\right] & \text { if } \xi \in D_{1}, \\ h_{2}(\xi) \cup e^{-1}\left[h_{2}(\xi)\right] & \text { if } \xi \in D_{2},\end{cases}
$$

and

$$
i_{q}(\{\xi, \eta\})= \begin{cases}i_{1}(\{\xi, \eta\}) & \text { if } \xi, \eta \in D_{1}, \\ i_{2}(\{\xi, \eta\}) & \text { if } \xi, \eta \in D_{2}, \\ f(\{\xi, \eta\}) \cap D_{q} & \text { otherwise. }\end{cases}
$$

Note that (A) implies that the set $i_{q}(\{\xi, \eta\})$ is well-defined for any $\xi, \eta \in D_{1} \cap D_{2}$, $\xi \neq \eta$; clearly $i_{q}$ is well-defined for the other pairs. Also, if $\xi \in D_{1} \cap D_{2}$, then the set $h_{q}(\xi)$ is well-defined because both of the conditions reduce to $h_{q}(\xi)=h_{1}(\xi) \cup h_{2}(\xi)$ by Lemmas 2.5 and 2.2(c). 
We have to show that $q \in \mathbb{P}_{f}$, i.e., that $q$ satisfies conditions 1,2 and 3 from Definition 1.2. The fact that $q$ satisfies conditions 1.2 , 1 and 1.2.3(c) follows directly from the definition of $q$ and from the fact that $p_{1}, p_{2} \in \mathbb{P}_{f}$. Condition $[1.22$ is satisfied because $p_{1}, p_{2} \in \mathbb{P}_{f}$ and the functions $e$ and $\delta_{2}$ are nondecreasing. In what follows we will be using Lemma 2.6 for $p=p_{1}, p_{2}$ and $g=\delta_{2}, e$, respectively. The hypothesis of the lemma is satisfied for these objects by 2.4(b) and 2.1(b).

Now we check conditions 1.23 (a) and (b). Let $\xi, \eta \in D_{q}, \xi<\eta$, and consider the following cases:

Case 1. $\xi, \eta \in D_{1}$.

It follows from the definition of $q$ and from Lemma 2.6 that

$$
\left(h_{q}(\xi) * h_{q}(\eta)\right) \cap D_{1}=h_{1}(\xi) * h_{1}(\eta)
$$

and

$$
\left(h_{q}(\xi) * h_{q}(\eta)\right) \cap\left(D_{2} \backslash D_{1}\right)=\delta_{2}^{-1}\left[h_{1}(\xi) * h_{1}(\eta)\right] \cap\left(D_{2} \backslash D_{1}\right) .
$$

Now let $\zeta \in h_{q}(\xi) * h_{q}(\eta)$.

Subcase 1.1. $\zeta \in D_{1}$.

In this subcase, $\zeta \in h_{1}(\xi) * h_{1}(\eta)$ and there is $\gamma \in i_{1}(\{\xi, \eta\})=i_{q}(\{\xi, \eta\})$ such that $\zeta \in h_{1}(\gamma) \subseteq h_{q}(\gamma)$, as we wanted.

Subcase 1.2. $\zeta \in D_{2} \backslash D_{1}$.

In this subcase, $\delta_{2}(\zeta) \in h_{1}(\xi) * h_{1}(\eta)$ and, since $\delta_{2}(\zeta), \xi, \eta \in D_{1}$ and $p_{1} \in \mathbb{P}_{f}$, there is $\gamma \in i_{1}(\{\xi, \eta\})=i_{q}(\{\xi, \eta\})$ such that $\delta_{2}(\zeta) \in h_{1}(\gamma)$. Since $\gamma \in D_{1}$, it follows by the definition of $q$ that $\zeta \in h_{q}(\gamma)$, as we wanted.

Case 2. $\xi, \eta \in D_{2}$.

It follows from the definition of $q$ and from Lemma 2.6 that

$$
\left(h_{q}(\xi) * h_{q}(\eta)\right) \cap D_{1}=h_{1}(\xi) * h_{1}(\eta)
$$

and

$$
\left(h_{q}(\xi) * h_{q}(\eta)\right) \cap\left(D_{2} \backslash D_{1}\right)=e^{-1}\left[h_{1}(\xi) * h_{1}(\eta)\right] \cap\left(D_{2} \backslash D_{1}\right) .
$$

Now let $\zeta \in h_{q}(\xi) * h_{q}(\eta)$.

Subcase 2.1. $\zeta \in D_{1} \backslash D_{2}$.

In this subcase, $e(\zeta) \in h_{2}(\xi) * h_{2}(\eta)$ and, since $e(\zeta), \xi, \eta \in D_{2}$ and $p_{2} \in \mathbb{P}_{f}$, there is $\gamma \in i_{2}(\{\xi, \eta\})=i_{q}(\{\xi, \eta\})$ such that $e(\zeta) \in h_{2}(\gamma)$. Since $\gamma \in D_{2}$, it follows by the definition of $q$ that $\zeta \in h_{q}(\gamma)$, as we wanted.

Subcase 2.2. $\zeta \in D_{2}$.

In this subcase, $\zeta \in h_{2}(\xi) * h_{2}(\eta)$ and there is $\gamma \in i_{2}(\{\xi, \eta\})=i_{q}(\{\xi, \eta\})$ such that $\zeta \in h_{2}(\gamma) \subseteq h_{q}(\gamma)$, as we wanted.

Case 3. $\xi \in D_{1} \backslash D_{2}$ and $\eta \in D_{2} \backslash D_{1}$.

Here we fix $\zeta \in h_{q}(\xi) * h_{q}(\eta)$ and we consider the following subcases:

Subcase 3.1. $\zeta \in D_{1}$.

In this subcase, $\zeta \in D_{1} \cap \xi \cap \eta$ and it follows from (B)(ii) that $\zeta \in f(\{\xi, \eta\})$. Hence, $\zeta \in D_{1} \cap f(\{\xi, \eta\}) \subseteq D_{q} \cap f(\{\xi, \eta\})=i_{q}(\{\xi, \eta\})$. Taking $\gamma=\zeta$, we conclude that $\zeta \in h_{q}(\gamma)$ and $\gamma \in i_{q}(\{\xi, \eta\})$, as we wanted. 
Subcase 3.2. $\zeta \in D_{2} \backslash D_{1}$.

First note that, regardless of the fact whether $h_{q}(\xi) * h_{q}(\eta)=h_{q}(\xi) \cap h_{q}(\eta)$ or $h_{q}(\xi) * h_{q}(\eta)=h_{q}(\xi) \backslash h_{q}(\eta)$, the assumption $\zeta \in h_{q}(\xi) * h_{q}(\eta)$ implies that $\zeta \in h_{q}(\xi)$.

In this subcase, it follows from the definition of $h_{q}(\xi)$ that $\delta_{2}(\zeta) \in h_{1}(\xi)$, so that $\delta_{2}(\zeta) \in D_{1} \cap \xi \cap \eta$, and it follows from (B)(ii) that $\delta_{2}(\zeta) \in f(\{\xi, \eta\}) \cap D_{q}=i_{q}(\{\xi, \eta\})$. By the definition of $\delta_{2}(\zeta), \zeta \in h_{2}\left(\delta_{2}(\zeta)\right) \subseteq h_{q}\left(\delta_{2}(\zeta)\right)$. Taking $\gamma=\delta_{2}(\zeta)$, we have that $\zeta \in h_{q}(\gamma)$ and $\gamma \in i_{q}(\{\xi, \eta\})$, concluding the proof of this subcase.

Case 4. $\xi \in D_{2} \backslash D_{1}$ and $\eta \in D_{1} \backslash D_{2}$.

Again we fix $\zeta \in h_{q}(\xi) * h_{q}(\eta)$ and we consider the following subcases:

Subcase 4.1. $\zeta \in D_{1}$.

The proof in this subcase follows identically to the proof of Subcase 3.1.

Subcase $4.21 \zeta \in D_{2} \backslash D_{1}$. We start this last subcase by proving the following:

Fact 1. $\{\zeta, \xi\} \cap \operatorname{dom}\left(\delta_{2}\right)$ is a nonempty set such that $\min \delta_{2}[\{\zeta, \xi\}]<\eta$ and if both $\zeta$ and $\xi$ are in $\operatorname{dom}\left(\delta_{2}\right)$, then $\delta_{2}(\zeta) \neq \delta_{2}(\xi)$.

Proof of Fact 1. First remark that, from the definition of $*$, it follows that if $\xi \notin$ $h_{q}(\eta)$, then $\zeta \in h_{q}(\xi) * h_{q}(\eta)=h_{q}(\xi) \cap h_{q}(\eta)$ and therefore $\zeta \in h_{q}(\eta)$. Analogously, if $\xi \in h_{q}(\eta)$, then $\zeta \in h_{q}(\xi) * h_{q}(\eta)=h_{q}(\xi) \backslash h_{q}(\eta)$ and therefore $\zeta \notin h_{q}(\eta)$. So,

$$
\left|\{\zeta, \xi\} \cap h_{q}(\eta)\right|=1 .
$$

From the definition of $q$ we have that, since $\xi, \zeta \notin D_{1}$ and $\eta \in D_{1}$ in this subcase, the above means that

$$
\left|\{\zeta, \xi\} \cap \delta_{2}^{-1}\left[h_{1}(\eta)\right]\right|=1,
$$

so that $\{\zeta, \xi\} \cap \operatorname{dom}\left(\delta_{2}\right)$ is a nonempty set. The above observation also implies that $\delta_{2}[\{\zeta, \xi\}] \cap h_{1}(\eta) \neq \emptyset$ and so, $\min \delta_{2}[\{\zeta, \xi\}] \leq \eta$. Now since $\eta \notin D_{2}$ and the range of $\delta_{2}$ is included in $D_{1} \cap D_{2}$, the inequality must be strict.

Finally, we have seen that $\zeta \in h_{q}(\eta)$ if and only if $\xi \notin h_{q}(\eta)$ and so, if both $\zeta$ and $\xi$ are in the domain of $\delta_{2}$, it follows that $\delta_{2}(\zeta) \in h_{1}(\eta)$ if and only if $\delta_{2}(\xi) \notin h_{1}(\eta)$, so that $\delta_{2}(\zeta) \neq \delta_{2}(\xi)$, concluding the proof of Fact 1 .

Take $\theta=\min \left\{\delta_{2}(\xi), \delta_{2}(\zeta)\right\}$ and note that $\theta \neq \xi$ since $\xi \in D_{2} \backslash D_{1}$ and the range of $\delta_{2}$ is included in $D_{1} \cap D_{2}$. We go now to the following subcases:

Subcase 4.2.1. $\theta<\xi$.

Here, $\theta \neq \delta_{2}(\xi)$ and therefore $\delta_{2}(\zeta)=\theta \in D_{1} \cap \xi \cap \eta$. From condition (B)(ii), it follows that $\delta_{2}(\zeta) \in f(\{\xi, \eta\}) \cap D_{q}=i_{q}(\{\xi, \eta\})$. Since $\zeta \in h_{2}\left(\delta_{2}(\zeta)\right) \subseteq h_{q}\left(\delta_{2}(\zeta)\right)$, taking $\gamma=\delta_{2}(\zeta)$, we have that $\zeta \in h_{q}(\gamma)$ and $\gamma \in i_{q}(\{\xi, \eta\})$, as we wanted.

Subcase 4.2.2. $\theta>\xi$.

Note that $\zeta \in h_{q}(\xi) * h_{q}(\eta) \subseteq h_{q}(\xi)$. Since $\zeta$ and $\xi$ satisfying the hypothesis of Case 4.2 are in $D_{2} \backslash D_{1}$, it follows from the definition of $h_{q}$ that $\zeta \in h_{2}(\xi)$. To finish, let us show the following:

Fact 2. $\zeta \in h_{2}(\xi) * h_{2}(\theta)$.

\footnotetext{
${ }^{1}$ This case is similar to Subcase 2.2 in the proof of Claim 2.7.2 of [1].
} 
Proof of Fact 2. First suppose $\theta=\delta_{2}(\xi)$. If $\zeta \notin \operatorname{dom}\left(\delta_{2}\right)$, then $\zeta \notin h_{2}\left(\delta_{2}(\xi)\right)$. If $\zeta \in \operatorname{dom}\left(\delta_{2}\right)$, from Fact 1 and the minimality of $\delta_{2}(\zeta)$, it follows that $\zeta \notin h_{2}\left(\delta_{2}(\xi)\right)$. Since $\xi \in h_{2}\left(\delta_{2}(\xi)\right)$, we have that $\zeta \in h_{2}(\xi) \backslash h_{2}\left(\delta_{2}(\xi)\right)=h_{2}(\xi) * h_{2}(\theta)$.

Now suppose $\theta=\delta_{2}(\zeta)$. Analogously we prove that $\xi \notin h_{2}\left(\delta_{2}(\zeta)\right)$ and $\zeta \in$ $h_{2}(\xi) \cap h_{2}\left(\delta_{2}(\zeta)\right)=h_{2}(\xi) * h_{2}(\theta)$, concluding the proof of Fact 2 .

Finally, since $p_{2} \in \mathbb{P}_{f}$, there is $\gamma \in i_{2}(\{\xi, \theta\})$ such that $\zeta \in h_{2}(\gamma) \subseteq h_{q}(\gamma)$. By condition (B)(i), which can be used by Fact 1 , we have that

$$
i_{2}(\{\xi, \theta\}) \subseteq f(\{\xi, \theta\}) \cap D_{2} \subseteq f(\{\xi, \eta\}) \cap D_{q}=i_{q}(\{\xi, \eta\}) .
$$

Hence, $\gamma \in i_{q}(\{\xi, \eta\})$ and $\zeta \in h_{q}(\gamma)$, concluding the proof of Subcase 4.2, Case 4 and thus concluding the proof of Claim 2.

Now that we know that $q \in \mathbb{P}_{f}$, let us check the other conclusions: it follows easily from the definition of $q$ and Lemma 2.5 that $q \leq p_{1}$ and analogously it follows from the definition of $q$ and Lemma 2.4 that $q \leq p_{2}$.

Finally, we verify the condition we want $q$ to satisfy, that is, $\xi \in h_{2}(\eta) \cup e^{-1}\left[h_{2}(\eta)\right]$ if and only if $e(\xi) \in h_{2}(\eta)$ : let $\xi \in D_{1}$ and $\eta \in D_{2}$ and consider again the following cases:

Case 1. $\xi \in D_{1} \cap D_{2}$.

It follows from the fact that in this case $e(\xi)=\xi$.

Case 2. $\xi \in D_{1} \backslash D_{2}$.

In this case, $\xi \in h_{2}(\eta) \cup e^{-1}\left[h_{2}(\eta)\right]$ if and only if $\xi \in e^{-1}\left[h_{2}(\eta)\right]$ if and only if $e(\xi) \in h_{2}(\eta)$, concluding the proof of the lemma.

\section{THE MAIN RESULTS}

To apply the key lemma proved in the previous section, the function $f$ on which the forcing $\mathbb{P}_{f}$ depends must satisfy a stronger version of the property $\Delta$ :

Definition 3.1. A function $f:\left[\omega_{2}\right]^{2} \rightarrow\left[\omega_{2}\right]^{\leq \omega}$ has the strong property $\Delta$ if $f(\{\xi, \eta\}) \subseteq \min \{\xi, \eta\}$ for all $\{\xi, \eta\} \in\left[\omega_{2}\right]^{2}$ and for any uncountable $\Delta$-system $\mathcal{A}$ of finite subsets of $\omega_{2}$, there are distinct $a, b \in \mathcal{A}$ and an order-preserving bijection $e: a \rightarrow b$ which is the identity on $a \cap b$ and such that $\xi \leq e(\xi)$ for all $\xi \in a$ and for any $\tau \in a \cap b$, any $\xi \in a \backslash b$ and any $\eta \in b \backslash a$ we have:

1) $a \cap \min \{\xi, \eta\} \subseteq f(\{\xi, \eta\})$

2) $\tau<\xi \Rightarrow f(\{\tau, \eta\}) \subseteq f(\{\xi, \eta\})$,

3) $\tau<\eta \Rightarrow f(\{\tau, \xi\}) \subseteq f(\{\xi, \eta\})$.

Finally we arrive at the main result of this paper.

Theorem 3.2. If $f:\left[\omega_{2}\right]^{2} \rightarrow\left[\omega_{2}\right] \leq \omega$ has the strong property $\Delta$, then $V^{\mathbb{P}_{f}}$ satisfies "for all $n \in \mathbb{N}, K_{f}^{n}$ is hereditarily separable".

Proof. We prove this by induction on $n \in \mathbb{N}$ : in $V^{\mathbb{P}_{f}}$, fix $n \in \mathbb{N}$ and suppose that for all $0 \leq i<n, K_{f}^{i}$ is hereditarily separable (take $K_{f}^{0}=\{*\}$ ) and let us show that $K_{f}^{n}$ is hereditarily separable. We will be using a well-known fact that a regular space is hereditarily separable if and only if it has no uncountable left-separated sequence (see Theorem 3.1 of [24]).

In $V$, suppose $\left(\dot{x}_{\alpha}\right)_{\alpha<\omega_{1}}$ is a sequence of names such that $\mathbb{P}_{f}$ forces that $\left(\dot{x}_{\alpha}\right)_{\alpha<\omega_{1}}$ is a left-separated sequence in $K_{f}^{n}$ of cardinality $\aleph_{1}$ and for each $\alpha<\omega_{1}$, we have that $\dot{x}_{\alpha}=\left(\dot{x}_{1}^{\alpha}, \ldots, \dot{x}_{n}^{\alpha}\right)$, where each $\dot{x}_{i}^{\alpha}$ is a name for an element of $K_{f}$. 
Notice that if

$$
\mathbb{P}_{f} \Vdash \exists 1 \leq i \leq n, \exists X \subseteq \omega_{1},|X|=\aleph_{1} \text { such that } \forall \alpha, \beta \in X, \dot{x}_{i}^{\alpha}=\dot{x}_{i}^{\beta},
$$

then

$\mathbb{P}_{f} \Vdash \exists 1 \leq i \leq n, \exists X \subseteq \omega_{1},|X|=\aleph_{1}$ such that $\left(\left(\dot{x}_{1}^{\alpha}, \ldots, \dot{x}_{i-1}^{\alpha}, \dot{x}_{i+1}^{\alpha}, \ldots, \dot{x}_{n}^{\alpha}\right)\right)_{\alpha \in X}$ is a left-separated sequence in $K_{f}^{n-1}$,

contradicting the inductive hypothesis. Therefore, we can assume without loss of generality that $\mathbb{P}_{f}$ forces that for all $1 \leq i \leq n$ and all $\alpha<\beta<\omega_{1}, \dot{x}_{i}^{\alpha} \neq{\dot{x_{i}}}^{\beta}$ and $\dot{x}_{i}^{\alpha} \in L_{f}=K_{f} \backslash\{*\}$.

By assertion $(+)$ following Definition 1.4, for each $\alpha<\omega_{1}$, there are names $\dot{F}_{1}^{\alpha}, \ldots, \dot{F}_{n}^{\alpha}$ for finite subsets of $\omega_{2}$ such that $\mathbb{P}_{f}$ forces that

$$
\forall \alpha<\omega_{1} \quad \forall 1 \leq i \leq n \quad \dot{x}_{i}^{\alpha} \in h\left(\dot{x}_{i}^{\alpha}\right) \backslash \bigcup_{\xi \in \dot{F}_{i}^{\alpha}} h(\xi)
$$

and

$$
\forall \alpha<\beta<\omega_{1} \quad \exists 1 \leq i \leq n \quad \dot{x}_{i}^{\alpha} \notin h\left(\dot{x}_{i}^{\beta}\right) \backslash \bigcup_{\xi \in \dot{F}_{i}^{\beta}} h(\xi) .
$$

For each $\alpha<\omega_{1}$, let $p_{\alpha}=\left(D_{\alpha}, h_{\alpha}, i_{\alpha}\right) \in \mathbb{P}_{f}, x_{1}^{\alpha}, \ldots, x_{n}^{\alpha} \in \omega_{2}$ and $F_{1}^{\alpha}, \ldots, F_{n}^{\alpha} \subseteq$ $\omega_{2}$ be finite such that

$$
p_{\alpha} \Vdash \forall 1 \leq i \leq n \quad \dot{x}_{i}^{\alpha}=\check{x}_{i}^{\alpha} \text { and } \dot{F}_{i}^{\alpha}=\check{F}_{i}^{\alpha} .
$$

By Lemma 2.2 of [11, we can assume without loss of generality that for all $\alpha<\omega_{1}$ and all $1 \leq i \leq n, F_{i}^{\alpha} \subseteq D_{\alpha}$ and $x_{i}^{\alpha} \in D_{\alpha}$.

By the $\Delta$-system Lemma, we can assume as well that $\left(D_{\alpha}\right)_{\alpha<\omega_{1}}$ forms a $\Delta$ system with root $D$. Since for each pair $\{\xi, \eta\} \subseteq D$ and each $\alpha<\omega_{1}$, we have that $i_{\alpha}(\{\xi, \eta\}) \in[f(\{\xi, \eta\})]^{<\omega}$, we may assume that for all $\alpha<\beta<\omega_{1}$, if $\xi, \eta \in D$, $\xi \neq \eta$, then $i_{\alpha}(\{\xi, \eta\})=i_{\beta}(\{\xi, \eta\})$.

By thinning out, we can assume without loss of generality that $\left(D_{\alpha}\right)_{\alpha<\omega_{1}}$ forms a $\Delta$-system with root $D$ such that for every $\alpha<\beta<\omega_{1}$ :

- $p_{\alpha}$ is isomorphic to $p_{\beta}$;

- $p_{\alpha}$ is lower than $p_{\beta}$;

- if $e_{\alpha \beta}: D_{\alpha} \rightarrow D_{\beta}$ is the order-preserving bijective function, then $e_{\alpha \beta}\left(x_{i}^{\alpha}\right)=$ $x_{i}^{\beta}$, for all $1 \leq i \leq n$.

Finally, we may assume that for all $1 \leq i \leq n$ we have: either $x_{i}^{\alpha}=x_{i}^{\beta}$ for all $\alpha<\beta<\omega_{1}$, or $x_{i}^{\alpha} \notin D$ for all $\alpha<\omega_{1}$, and actually the second case holds by our initial assumption about the sequence.

Since $f$ has the strong property $\Delta$, there are $\alpha<\beta<\omega_{1}$ such that for all $\zeta \in D$, all $\xi \in D_{\alpha} \backslash D$ and all $\eta \in D_{\beta} \backslash D$ :

(i) $D_{\alpha} \cap \xi \cap \eta \subseteq f(\{\xi, \eta\})$;

(ii) if $\zeta<\xi$, then $f(\{\zeta, \eta\}) \subseteq f(\{\xi, \eta\})$;

(iii) if $\zeta<\eta$, then $f(\{\zeta, \xi\}) \subseteq f(\{\xi, \eta\})$.

Note that $p_{\alpha}$ and $p_{\beta}$ satisfy the hypothesis of Lemma 2.7. Hence, there is $q \leq p_{\alpha}, p_{\beta}$ in $\mathbb{P}_{f}$ such that for all $\xi \in D_{\alpha}$ and all $\eta \in D_{\beta}$,

$$
\xi \in h_{q}(\eta) \text { if and only if } e_{\alpha \beta}(\xi) \in h_{p_{\beta}}(\eta) \text {. }
$$

Then, for all $1 \leq i \leq n$ and all $\xi \in D_{\beta}$, we have that

$$
x_{i}^{\alpha} \in h_{q}(\xi) \text { if and only if } x_{i}^{\beta} \in h_{p_{\beta}}(\xi) .
$$


So we have that

$$
x_{i}^{\alpha} \in h_{q}\left(x_{i}^{\beta}\right) \backslash \bigcup_{\xi \in F_{i}^{\beta}} h_{q}(\xi) .
$$

But $q \leq p_{\alpha}, p_{\beta}$ and then

$$
q \Vdash \forall 1 \leq i \leq n, \dot{x}_{i}^{\alpha}=\check{x}_{i}^{\alpha}, \dot{x}_{i}^{\beta}=\check{x}_{i}^{\beta} \text { and } \dot{F}_{i}^{\beta}=\check{F}_{i}^{\beta} .
$$

Therefore,

$$
q \Vdash \forall 1 \leq i \leq n, \dot{x}_{i}^{\alpha}=\check{x}_{i}^{\alpha} \in h\left(\check{x}_{i}^{\beta}\right) \backslash \bigcup_{\xi \in \check{F}_{i}^{\beta}} h(\xi)=h\left(\dot{x}_{i}^{\beta}\right) \backslash \bigcup_{\xi \in \dot{F}_{i}^{\beta}} h(\xi),
$$

contradicting the hypothesis about $\dot{x}_{i}^{\alpha}, \dot{x}_{i}^{\beta}$ and $\dot{F}_{i}^{\beta}$.

Corollary 3.3. It is relatively consistent with ZFC that there is a hereditarily separable compact scattered space of height $\omega_{2}$.

Proof. Since each level of the Cantor-Bendixson decomposition of $K_{f}$ is a discrete subset of $K_{f}$, it follows that every level of it is countable. But $\left|K_{f}\right|=\aleph_{2}$ and $K_{f}=$ $\bigcup_{\alpha<h t\left(K_{f}\right)} K_{f}^{(\alpha)} \backslash K_{f}^{(\alpha+1)}$, so that $h t\left(K_{f}\right) \geq \omega_{2}$. It is easy to see that $\bigcap_{\alpha<\omega_{2}} K_{f}^{(\alpha)}=$ $\{*\}$ concluding that $h t\left(K_{f}\right)=\omega_{2}$.

Corollary 3.4. It is relatively consistent with ZFC that there is a hereditarily separable compact space with hereditary Lindelof degree equal to $\aleph_{2}$. In particular, it is relatively consistent with $Z F C$ that there is a compact space $K$ such that $h L(K) \not \leq$ $h d(K)^{+}$.

Proof. It follows from the fact that $h L\left(K_{f}\right) \leq\left|K_{f}\right|=\aleph_{2}$ and that $\left\{K_{f} \backslash K_{f}^{(\alpha)}: \alpha<\right.$ $\left.\omega_{2}\right\}$ is an open covering of $K_{f} \backslash\{*\}$ which does not admit a subcovering of strictly smaller cardinality.

Corollary 3.5. It is relatively consistent with $Z F C$ that there is an Asplund space $C(K)$ of density $\aleph_{2}$ which does not admit any Fréchet smooth renorming and which does not contain an uncountable biorthogonal system.

Proof. Since every finite power of $K_{f}$ is hereditarily separable, Lemma 4.37 and Theorem 4.38 of [6] imply that $C\left(K_{f}\right)$ is hereditarily Lindelöf relative to its pointwise convergence topology. But for compact scattered spaces $K$, the pointwise convergence topology and the weak topology of $C(K)$ coincide (see Theorem 7.4 of 20]), so that $C\left(K_{f}\right)$ is hereditarily Lindelöf relative to its weak topology.

Now, if $C\left(K_{f}\right)$ admits a Fréchet smooth renorming, by Corollaries 8.34 (due to Mazur [18]) and 8.36 of [6] (due to Jiménez Sevilla and Moreno [10]) it contains an uncountable bounded subset $A$ such that for every $x_{0} \in A, x_{0}$ is not in the (norm-) closed convex hull of $A \backslash\left\{x_{0}\right\}$, that is, $x_{0} \notin \overline{\operatorname{conv}}\left(A \backslash\left\{x_{0}\right\}\right)$. Since the weak and norm convex closures coincide in Banach spaces, $A$ turns out to be an uncountable discrete family of $C\left(K_{f}\right)$ relative to its weak topology, which contradicts the fact that $C\left(K_{f}\right)$ is hereditarily Lindelöf relative to its weak topology.

Now, if $C\left(K_{f}\right)$ admits an uncountable biorthogonal system $\left(x_{\alpha}, \varphi_{\alpha}\right)_{\alpha<\omega_{1}} \subseteq$ $C\left(K_{f}\right) \times C\left(K_{f}\right)^{*}$, then $\left\{x_{\alpha}: \alpha<\omega_{1}\right\}$ is an uncountable discrete family of $C\left(K_{f}\right)$ relative to its weak topology, contradicting the fact that $C\left(K_{f}\right)$ is hereditarily Lindelöf relative to its weak topology. 
One should compare the above corollary to Theorem 4.41 of [6] (due to Ostaszewski [21]) and to Corollary 8.37 of [6] (due to Jiménez Sevilla and Moreno [10]).

\section{The existence of the ReQuired Function $f$}

In this section we prove the consistency of the existence of a function with the strong property $\Delta$. It turns out that we are even able to prove the consistency of the existence of such a function with its range included in the family of finite (rather than countable) subsets of $\omega_{2}$. The method is quite involved but, as shown at the end of this section, forcings preserving $\mathrm{CH}$ (as in [2]) cannot serve for this purpose even if we were interested in a function with its range included in countable subsets of $\omega_{2}$.

4.1. Forcing with side conditions in Velleman's simplified morasses. To construct a forcing which adds the required auxiliary function on pairs of $\omega_{2}$ we will need a family of countable subsets of $\omega_{2}$ with some strong properties. The following proposition establishes a list of the most useful properties:

Proposition 4.1. It is relatively consistent with $\mathrm{ZFC}+\mathrm{CH}$ that there exists a family $\mathcal{F} \subseteq\left[\omega_{2}\right]^{\omega}$ which satisfies the following properties:

1) $(\mathcal{F}, \subseteq)$ is well-founded (thus, one can talk about $\operatorname{rank}(X)$ for $X \in \mathcal{F})$.

2) $\mathcal{F}$ is stationary in $\left[\omega_{2}\right]^{\omega}$ (see [1]).

3) If $\alpha \in X, Y \in \mathcal{F}$ and $\operatorname{rank}(X) \leq \operatorname{rank}(Y)$, then $X \cap \alpha \subseteq Y \cap \alpha$.

If $M$ is a countable elementary submodel of $H\left(\omega_{3}\right)$ containing $\omega_{1}, \omega_{2}, \mathcal{F}$ and $X=$ $M \cap \omega_{2} \in \mathcal{F}$, then

4) $M \cap \omega_{1}=\operatorname{rank}(X)$.

5) $Y \subset X, Y \in \mathcal{F}$ implies $Y \in M$.

6) $X_{1}, \ldots, X_{n} \in \mathcal{F}$ for $n \in N$ and $\operatorname{rank}\left(X_{i}\right)<\operatorname{rank}(X)$ for $1 \leq i \leq n$ implies that there is $Z \in \mathcal{F}$ such that $Z \in M$ and $X \cap\left(X_{1} \cup \ldots \cup X_{n}\right) \subseteq Z$.

Proof. We will prove that a simplified Velleman's $\left(\omega_{1}, 1\right)$-morass (see [30] which is a stationary coding set (see [31]) satisfies the above properties. The proof relies heavily on the properties of Velleman's morasses obtained in [15. We will often refer to this paper; in particular, we adopt definitions of simplified morass and stationary coding set from this paper (Section 2). The consistency of the existence of such morasses can be immediately obtained from the corresponding proof for semimorasses in 14, Theorem 3, Section 2.

1) follows from Definition 2.1 of [15] and 2) from the fact that $\mathcal{F}$ is assumed to be a stationary coding set. To prove 3 ) apply 2.5 of [15]. Now 4) is Fact 2.7 of [15] and 5) is Fact 2.6 of [15]. To obtain 6) apply Fact 2.8 of [15] to each $X_{i}$ obtaining $Z\left(X_{i}\right)$ such that $Z\left(X_{i}\right) \in M \cap \mathcal{F}$ and $X_{i} \cap X \subseteq Z\left(X_{i}\right)$. Now use the elementarity of $M$ and the directedness of $\mathcal{F}$ (see Definition 2.1 of [15]) to obtain $Z$ as in 6).

Now we will adopt a few facts from [16] and [15] concerning forcing with side conditions in $\mathcal{F}$. As explained in these papers, to use elements of $\mathcal{F}$ as side conditions means to use forcings $P$ whose conditions are of the form $(p, A)$ where $p$ is a finite condition of a natural forcing adding the structure in question and $A$ is a finite subset of $\mathcal{F}$. This is like using models as side conditions in the method of forcing with models as side conditions developed by Todorcevic (see [27]). The order is given by 
the forcing order on the first coordinate and inverse inclusion on the second coordinate. In addition we require the existence of some natural projections of $p$ onto the elements of $A$ as a part of the definition of the forcing notion. The properties 1) - 6) above allow us to perform many maneuvers with ease; also the definitions are simpler. This method appears to be equivalent to the variant of Todorcevic's method where one employs matrices of models (see [28, Section 4, for an example with detailed definitions). The price we need to pay for this convenience is that $P$ is not proper (unlike Todorcevic's forcings,) but only $\mathcal{F}$-proper, i.e., there is a club $\mathcal{C} \subseteq\left[\omega_{2}\right]^{\omega}$ such that for models $M \prec H\left(\omega_{3}\right)$ such that $M \in \mathcal{F} \cap \mathcal{C}$ and $p \in P \cap M$, there are $(P, M)$-generic conditions stronger than $p$. As $\mathcal{F}$ may be assumed to be stationary, $\mathcal{F}$-properness implies the preservation of $\omega_{1}$ (for a proof as for proper forcings, see [1]). The preservation of bigger cardinals follows from the $\omega_{2}$-chain condition. Note that the fact that the forcing is not proper but preserves cardinals is no limitation in the applications that one seeks here, i.e., consistent existence of structures of sizes bigger than $\omega_{1}$. Let us describe basic notions related to forcing with side conditions in $\mathcal{F}$ that we will use.

Definition 4.2. Suppose $\mathcal{F} \subseteq\left[\omega_{2}\right]^{\omega}$. We say that a forcing notion $P$ is $\mathcal{F}$-proper if there is $\theta>\left(2^{|P|}\right)^{+}$and a club set $\mathcal{C} \subseteq[H(\theta)]^{\omega}$ such that whenever $p \in M \in \mathcal{C}$ and $M \cap \omega_{2} \in \mathcal{F}$, then there is a $(P, M)$-generic $p_{0} \leq p$; i.e., $D \cap M$ is predense below $p_{0}$ for every $D \in M$ which is dense in $P$.

Fact 4.3. Suppose $\mathcal{F} \subseteq\left[\omega_{2}\right]^{\omega}$ is a stationary set and $P$ is an $\mathcal{F}$-proper forcing notion. Then $P$ preserves $\omega_{1}$.

Proof. The proof is a straightforward version of Shelah's paradigmatic proof of the preservation of $\omega_{1}$ by proper forcings (see [26] or [1]).

The following definition and lemmas are formulations of well-known techniques (originated in Shelah's use of elementary submodels in forcing) and will simplify our further arguments.

Definition 4.4. Let $P$ be a notion of forcing, $q \in P$ and let $\theta>\left(2^{|P|}\right)^{+}$. Suppose $M \prec H(\theta)$ and $P, \pi_{1}, \ldots, \pi_{k} \in M$. We say that a formula $\phi\left(x_{0}, x_{1}, \ldots, x_{k}\right)$ well reflects $q$ in $\left(M ; \pi_{1}, \ldots, \pi_{k}\right)$ whenever the following are satisfied:

i) $\phi\left(q, \pi_{1}, \ldots, \pi_{k}\right)$ holds in $H(\theta)$;

ii) whenever $s \in M$ is such that $\phi\left(s, \pi_{1}, \ldots, \pi_{k}\right)$ holds in $M$, then $q$ and $s$ are compatible.

Definition 4.5. Suppose $\mathcal{F} \subseteq\left[\omega_{2}\right]^{\omega}$ and suppose $P$ is a notion of forcing. We say that $P$ is simply $\mathcal{F}$-proper if there is $\theta$ such that whenever

a) $p \in P$,

b) $M \prec H(\theta), M$ countable,

c) $p, P, \mathcal{F} \in M$,

d) $M \cap \omega_{2} \in \mathcal{F}$

then there is $p_{0} \leq p$ such that if $q \geq p_{0}$, then there are $\pi_{1}, \ldots, \pi_{k} \in M$ and a formula $\phi\left(x_{0}, x_{1}, \ldots, x_{k}\right)$ which well reflects $q$ in $\left(M, \pi_{1}, \ldots, \pi_{k}\right)$.

Lemma 4.6. If $P$ is simply $\mathcal{F}$-proper, then $P$ is $\mathcal{F}$-proper.

Proof. We will prove that whenever $M, p$ are as in a) - d) of Definition 4.5, then $p_{0}$ is a $(P, M)$-generic condition. Letting $D \in M$ be dense, we will show that $D \cap M$ 
is predense below $p_{0}$. Letting $q \leq p_{0}$, we may w.l.o.g. assume that $q \in D$. Let $\pi_{1}, \ldots, \pi_{k} \in M$ and $\phi\left(x_{0}, x_{1}, \ldots, x_{k}\right)$ be such that $\phi\left(x_{0}, x_{1}, \ldots, x_{k}\right)$ well reflects $q$ in $\left(M, \pi_{1}, \ldots, \pi_{k}\right)$. By i) of Definition 4.4 we have that $\phi\left(q, \pi_{1}, \ldots \pi_{k}\right)$ in $H(\theta)$. By its elementarity, $M$ satisfies the formula " $\exists x \in P \phi\left(x, \pi_{1}, \ldots \pi_{k}\right) \& x \in D$ ". So let $s \in M$ witness this fact. Now by Definition 4.4ii), $s$ and $q$ are compatible, so $D \cap M$ contains a condition compatible with $q$, which proves that $D \cap M$ is predense below $q$, which completes the proof.

4.2. Adding a function with the strong property $\Delta$. Fix a family $\mathcal{F} \subseteq\left[\omega_{2}\right]^{\omega}$ satisfying 1) - 6) of Proposition 4.1. We will assume familiarity of the reader with elementary submodels of structures $H(\theta)$. In particular we will make use of facts such as that countable elements of such models are their subsets or that such models contain $\omega$. See [5] for more on this subject. We consider the following forcing $P$ whose conditions $p$ are of the form: $p=\left(a_{p}, f_{p}, A_{p}\right)$, where

a) $a_{p} \in\left[\omega_{2}\right]^{<\omega}$;

b) $f_{p}:\left[a_{p}\right]^{2} \rightarrow\left[\omega_{2}\right]^{<\omega}$

c) $A_{p} \in[\mathcal{F}]^{<\omega}$

d) $f_{p}(\alpha, \beta) \subseteq \bigcap\left\{X: X \in A_{p}, \alpha, \beta \in X\right\} \cap \min \{\alpha, \beta\}$ for any distinct $\alpha, \beta \in a_{p}$.

The order is just the inverse inclusion, i.e., $p \leq q$ if and only if $a_{p} \supseteq a_{q}, f_{p} \supseteq f_{q}$, $A_{p} \supseteq A_{q}$.

Fact 4.7. $P$ is simply $\mathcal{F}$-proper.

Proof. Let $\theta=\omega_{3}$ and let $M$ and $p$ be as in a) - d) of Definition 4.5. The existence of such an $M$ follows from the stationarity of $\mathcal{F}$. Let $X_{0}=M \cap \omega_{2}$. Let $p_{0}=$ $\left(a_{p}, f_{p}, A_{p} \cup\left\{X_{0}\right\}\right)$. Finally let $q \leq p_{0}$. The proof consists of using Lemma 4.6 and finding the parameters $\pi_{1}, \ldots, \pi_{k} \in M$ and a formula $\phi\left(x_{0}, x_{1}, \ldots, x_{k}\right)$ which well reflects $q$ in $\left(M, \pi_{1}, \ldots, \pi_{k}\right)$.

Define $q \mid M=\left(a_{q} \cap M, f_{q} \mid M, A_{q} \cap M\right)$. Introduce the notation $\delta=M \cap \omega_{1}=$ $\operatorname{rank}(M)$, where the second equality follows from 4) of Proposition 4.1. Note that $A_{q} \cap M=A_{q \mid M}=\left\{X \in A_{q}: X \subset X_{0}\right\}$. This follows from 5) of Proposition 4.1. The fact that $[M]^{<\omega} \subseteq M$ implies that $a_{q \mid M}, A_{q \mid M} \in M$. Also, as d) of the definition of the forcing is satisfied for $q$ and $\alpha, \beta \in a_{q}$, we have that $f_{q}(\alpha, \beta) \subseteq X_{0}=M \cap \omega_{2}$ for $\alpha, \beta \in a_{q} \cap X_{0}$. So, we may conclude that $f_{q \mid M} \in M$; in other words, we have $q \mid M \in M \cap P$. It is clear that $q \mid M \leq p$. By 6) of Proposition 4.1 and the fact that $[M]^{<\omega} \subseteq M$, in $M$ there is a $Z \in \mathcal{F}$ such that $\bigcup\{X \cap M: \operatorname{rank}(X)<\delta, X \in$ $\left.A_{q}\right\} \subseteq Z$. Let $\phi\left(x_{0}, x_{1}, x_{2}, x_{3}, x_{4}\right)$ be the formula which says that $x_{0}$ is a condition of the partial order $x_{4}$ which extends in $x_{4}$ the condition $x_{3}$ and such that the difference between the first coordinate of $x_{0}$ and $x_{2}$ is disjoint from $x_{1}$.

Claim. $\phi\left(x_{0}, x_{1}, x_{2}, x_{3}, x_{4}\right)$ well reflects $q$ in $\left(M, Z, a_{q \mid M}, q \mid M, P\right)$.

Proof of the Claim. It is clear that $\phi\left(q, Z, a_{q \mid M}, q \mid M, P\right)$ holds in $H\left(\omega_{3}\right)$. Now let $s \in M$ be a condition satisfying $\phi\left(s, Z, a_{q \mid M}, q \mid M, P\right)$; i.e., $s$ extends in $P$ the condition $q \mid M$ and $a_{s} \backslash a_{q \mid M}$ is disjoint from $Z$. Define the common extension $r$ of $q$ and $s$ as follows: $a_{r}=a_{s} \cup a_{q}, f_{r}=f_{s} \cup f_{q} \cup h, A_{r}=A_{s} \cup A_{q}$, where $h(\{\alpha, \beta\})=\emptyset$ for $\{\alpha, \beta\} \in\left[a_{s} \cup a_{q}\right]^{2}-\left(\left[a_{s}\right]^{2} \cup\left[a_{q}\right]^{2}\right)$. Such an $f_{r}$ is a function on $\left[a_{r}\right]^{2}$ since $q \mid M \geq q, s$. Clearly all clauses of the definition of the forcing $P$ but d) are trivially satisfied by $r$. So let us prove d). Letting $\alpha, \beta \in a_{r}$ and $X \in A_{r}$, we will consider a few cases. 
Case 1. $\alpha, \beta \in a_{s}, X \in A_{s}$.

This is trivial because $s \in P$.

Case 2. $\alpha, \beta \in a_{q}, X \in A_{q}$.

This is trivial because $q \in P$.

Case 3. $\alpha, \beta \in a_{s}, X \in A_{q}$.

Since $\phi\left(s, Z, a_{q \mid M}, q \mid M, P\right)$ holds in $M$ we have that either $\operatorname{rank}(X) \geq \delta=$ $\operatorname{rank}\left(M \cap \omega_{2}\right)=\operatorname{rank}\left(X_{0}\right)$, in which case d) is satisfied because $f_{r}(\{\alpha, \beta\})=$ $f_{s}(\{\alpha, \beta\}) \subseteq X_{0} \cap \min \{\alpha, \beta\} \subseteq X \cap \min \{\alpha, \beta\}$ by d) for $s$ and 3) of Proposition 4.1 or $\operatorname{rank}(X)<\delta$ and then by the definition of $\phi$ and $Z$ we get that $\alpha, \beta \in a_{s} \cap a_{q}$, so we are again in Case 2.

Case 4. $\alpha, \beta \in a_{q}, X \in A_{s}$.

This means that $\alpha, \beta \in M$, because $s \in M$, i.e., $\alpha, \beta \in a_{s} \cap a_{q}$, so we are again in Case 1.

Case 5. $\alpha \in a_{s} \backslash a_{q}$ and $\beta \in a_{q} \backslash a_{s}$.

Then $h(\{\alpha, \beta\})=\emptyset$.

The proof of the claim completes the proof of Fact 4.7.

Definition 4.8. For $p \in P$, call the set $a_{p} \cup f\left[\left[a_{p}\right]^{2}\right] \cup \bigcup A_{p}$ the support of $p$ and denote it by $\operatorname{supp}(p)$.

Definition 4.9. We say that two conditions $p, q$ of $P$ are isomorphic (via $\pi$ : $\operatorname{supp}(p) \rightarrow \operatorname{supp}(q))$ if $\pi: \operatorname{supp}(p) \rightarrow \operatorname{supp}(q)$ is an order-preserving bijection constant on $\operatorname{supp}(p) \cap \operatorname{supp}(q)$ and

i) $\pi\left[a_{p}\right]=a_{q}$;

ii) $\left\{\pi[X]: X \in A_{p}\right\}=A_{q}$;

iii) $f_{q}(\{\pi(\alpha), \pi(\beta)\})=\pi\left[f_{p}(\{\alpha, \beta\})\right]$ for all $\alpha, \beta \in a_{p}$.

Lemma 4.10. Suppose $p, q \in P$ are isomorphic via $\pi: \operatorname{supp}(p) \rightarrow \operatorname{supp}(q)$. Then they are compatible.

Proof. Define the common extension $r$ of $p$ and $q$ as follows: $a_{r}=a_{p} \cup a_{q}, f_{r}=$ $f_{p} \cup f_{q} \cup h, A_{r}=A_{p} \cup A_{q}$, where $h(\{\alpha, \beta\})=\emptyset$ for $\{\alpha, \beta\} \in\left[a_{p} \cup a_{q}\right]^{2}-\left(\left[a_{p}\right]^{2} \cup\left[a_{q}\right]^{2}\right)$. The only nonautomatic condition of the definition of $P$ which needs to be checked is d).

Case 1. $\alpha, \beta \in a_{r}$.

If $X \in A_{r}$, we are trivially done. If $X \in A_{q}$ and $\alpha, \beta \in X$, then $\alpha, \beta \in$ $\operatorname{supp}(p) \cap \operatorname{supp}(q)$; hence $\alpha, \beta \in a_{p} \cap a_{q}$ and hence again use d) for $q$.

Case 2. $\alpha, \beta \in a_{q}$.

This is similar to the previous case.

Case 3. $\alpha \in a_{r} \backslash a_{q}, \beta \in a_{q} \backslash a_{r}$.

In this case $h$ is empty.

Fact 4.11. Assuming $\mathrm{CH}$, the forcing $P$ is $\omega_{2}$-c.c. Thus by Fact 4.7, Lemma 4.6 and Fact 4.3, $P$ preserves cardinals.

Proof. By the previous lemma the proof is a standard application of the $\Delta$-system lemma to the sequence of supports $\left\{\operatorname{supp}\left(p_{\xi}\right): \xi<\omega_{2}\right\}$ of some conditions $p_{\xi} \in P$ under our cardinal arithmetic assumption. 
Theorem 4.12. In $V^{P}$ there is a function $f:\left[\omega_{2}\right]^{2} \rightarrow\left[\omega_{2}\right]^{<\omega}$ with the strong property $\Delta$.

Proof. Clearly, we claim that $f=\bigcup\left\{f_{p}: p \in G\right\}$ defines such a function, where $G$ is a $P$-generic over $V$. Let $\dot{f}$ be a name for it.

Fix a set $A=\left\{\dot{a}^{\alpha}: \alpha<\omega_{1}\right\}$ of $P$-names for elements of an uncountable $\Delta$-system of $n$-tuples $\dot{a}=\left\{\dot{a}_{i}: i<n\right\}$ of elements of $\omega_{2}$ for which there are bijections $e$ as in Definition 3.1 (any uncountable $\Delta$-system has such an uncountable subsystem). Fix a condition $p \in P$.

Take a model $M \prec H\left(\omega_{3}\right)$ such that $M \cap \omega_{2}=X_{0} \in \mathcal{F}$ and $p \in P \cap M ; \mathcal{F} \in M$ and $\left\{\dot{a}^{\alpha}: \alpha<\omega_{1}\right\} \in M$. We will show that there are $\alpha_{1}<\alpha_{2}<\omega_{1}$ and $r \leq p$ such that $r$ forces 1), 2), 3) of Definition 3.1 for $\dot{a}^{\alpha_{1}}$ and $\dot{a}^{\alpha_{2}}$.

First take a condition $p_{0} \leq p$ as in Fact 4.7 i.e., $a_{p_{0}}=a_{p}, f_{p_{0}}=f_{p}, A_{p_{0}}=A_{p} \cup$ $X_{0}$. Take $q \leq p_{0}$ and $\alpha_{1} \in \omega_{1}$ such that there is $b$ such that $b \backslash M \neq \emptyset, q \Vdash \dot{a}^{\alpha_{1}}=\check{b}$ and $b \subseteq a_{q}$. This can be done as $\left\{\dot{a}^{\alpha}: \alpha<\omega_{1}\right\}$ is a sequence of names for an uncountable $\Delta$-system of sets and $|M|=\omega$. Proceed as in the proof of Fact 4.7 i.e., choose $Z$ and $\phi$ as in Fact 4.7 So, we have $\phi\left(q, Z, a_{q \mid M}, q \mid M, P\right)$ in $H\left(\omega_{3}\right)$ and so by the elementarity of $M$, we can find an $s$ and $\alpha_{2}$ such that $\phi\left(s, Z, a_{q \mid M}, q \mid M, P\right)$ holds in $M$ and moreover there is $a$ such that $a \backslash(b \cap M) \in[M \backslash Z]^{<\omega}$ and such that $s \Vdash \dot{a}^{\alpha_{2}}=\check{a}$ and $a \subseteq a_{s}$. Now we will obtain another amalgamation $r$ of $s$ and $q$ which will force 1), 2) and 3) of Definition 3.1. Let $a_{r}=a_{s} \cup a_{q}, f_{r}=f_{s} \cup f_{q} \cup h$. For $\xi \in a_{s} \backslash a_{q}$ and $\eta \in a_{q} \backslash a_{s}$ :

$$
h(\{\xi, \eta\})=[A \cup B \cup C] \cap D,
$$

where

$$
\begin{gathered}
A=a \cap \min \{\xi, \eta\}, \\
B=\bigcup\left\{f_{s}(\{\tau, \xi\}): \tau \in a \cap b, \tau<\eta\right\}, \\
C=\bigcup\left\{f_{q}(\{\tau, \eta\}): \tau \in a \cap b, \tau<\xi\right\}, \\
D=\min \{\xi, \eta\} \cap \bigcap\left\{X \in A_{q}: \xi, \eta \in X, \operatorname{rank}(X) \geq \delta\right\} .
\end{gathered}
$$

First let us check that $r$ is a common extension of $q$ and $s$. The proof also follows the cases as in the Claim in the proof of Fact 4.7 All are checked in the same manner except for Case 5 where one may assume that $X \in A_{q}$ as $\beta \notin M$. This time the inclusion in the set $\mathrm{D}$ guarantees that d) holds in Case 5.

Now we will check 1), 2) and 3) of Definition 3.1 for $a, b$ as above and $f_{r}$. This will be enough since $r \Vdash \dot{a}^{\alpha_{1}}=\check{b}, \dot{a}^{\alpha_{2}}=\check{a}$ and $r \Vdash f_{r} \subseteq \dot{f}$. Suppose $\xi \in a \backslash b$ and $\eta \in b \backslash a$. By the form of the definition of $f_{r}(\{\xi, \eta\})=h(\{\xi, \eta\})$ it will be enough to prove that the sets $A, B$ and $C$ are actually included in $\min \{\xi, \eta\} \cap X$ for any $X \in A_{q}$ such that $\operatorname{rank}(X) \geq \delta$ and $\xi, \eta \in X$. So, let $X \in A_{q}$ be any element such that $\operatorname{rank}(X) \geq \delta$ and $\xi, \eta \in X$.

For 1) of Definition 3.1, note that since $X_{0}=M \cap \omega_{2}$ and $\operatorname{rank}\left(X_{0}\right)=\delta$ we have that $M \cap \min \{\xi, \eta\}$ is included in $X$ by 3) of Proposition 4.1. Hence, as $a \subseteq M$, we have $a \cap \min \{\xi, \eta\} \subseteq \min \{\xi, \eta\} \cap X$; that is, we obtain 1).

To get 2) of Definition 3.1 assume that $\tau \in a \cap b$ and $\tau<\xi$; hence $\min \{\tau, \eta\} \leq$ $\min \{\xi, \eta\}$. Note again, by 3) of Proposition 4.1, that $M \cap \xi \subseteq X \cap \xi$, which implies in this case that $\tau \in X$. Hence, since $\tau, \eta \in a_{q}$, by d) of the definition of the forcing, we have that $f_{q}(\{\tau, \eta\}) \subseteq X \cap \min \{\tau, \eta\} \subseteq X \cap \min \{\xi, \eta\}$, so we obtain 2). 
To get 3) of Definition 3.1 assume that $\tau \in a \cap b$ and $\tau<\eta$; hence $\min \{\tau, \xi\} \leq$ $\min \{\xi, \eta\}$. We have that $\tau, \xi \in M \cap \xi$, and again $M \cap \xi \subseteq X$. Hence, since $\xi, \tau \in a_{s}$, by d) of the definition of the forcing, and the fact that $s \in M$, we have that $f_{s}(\{\tau, \xi\}) \cap \min \{\tau, \xi\} \subseteq X \cap \min \{\tau, \xi\} \subseteq X \cap \min \{\xi, \eta\}$, so we obtain 3).

Remark 4.13. For any $k \leq \omega$ and any uncountable $\Delta$-system one can have $k$ sets satisfying 1), 2) and 3) of Definition 3.1 This follows from the Dushnik-Miller theorem; see [9, Theorem 9.7.

4.3. $\mathbf{C H}$ and the strong property $\Delta$. In this section we prove that $\mathrm{CH}$ implies that there is no function $f$ such as in the previous section, even if we allow $f$ to take countable sets as values. This also proves that the strong property $\Delta$ cannot be obtained as in Baumgartner and Shelah [2, that is, by a forcing which preserves $\mathrm{CH}$.

Proposition 4.14. (CH) There is no $f:\left[\omega_{2}\right]^{2} \rightarrow\left[\omega_{2}\right]^{\omega}$ such that for every $\Delta$ system $\mathcal{A}$ of finite subsets of $\omega_{2}$ of cardinality $\aleph_{1}$, there exist distinct $a, b \in \mathcal{A}$ such that

$$
\forall \xi \in a \backslash b \quad \forall \eta \in b \backslash a \quad a \cap \xi \cap \eta \subseteq f(\{\xi, \eta\}) .
$$

Proof. Suppose that $f:\left[\omega_{2}\right]^{2} \rightarrow\left[\omega_{2}\right]^{\omega}$. For an $A \subseteq \omega_{2}$ and $\xi \in \omega_{2} \backslash A$ define

$$
f_{A, \xi}: A \rightarrow[A]^{\omega}, \quad f_{A, \xi}(\eta)=f(\{\eta, \xi\}) \cap A \quad \forall \eta \in A .
$$

Let $M \prec H\left(\omega_{3}\right)$ be closed under its countable subsets (here we use CH) $|M|=\omega_{1}$, $\omega_{1} \subseteq M ; \omega_{1}, \omega_{2}, f \in M$ and such that $\sup \left(M \cap \omega_{2}\right)=\gamma$ has an uncountable cofinality.

By recursion construct a sequence $\left(\alpha_{\xi}, \beta_{\xi}\right)_{\xi<\omega_{1}}$ which satisfies:

1) $\omega_{1}<\alpha_{\xi}, \beta_{\xi} \in M \cap \gamma$;

2) $\alpha_{\xi}<\beta_{\xi}<\alpha_{\eta}$ for all $\xi<\eta$;

3) $f_{A_{\eta}, \beta_{\eta}}=f_{A_{\eta}, \gamma}$, where $A_{\eta}=\left\{\alpha_{\xi}, \beta_{\xi}: \xi<\eta\right\}$;

4) $\alpha_{\eta} \notin f\left(\left\{\beta_{\eta}, \gamma\right\}\right)$.

To justify that this construction can be carried out assume that we have $A_{\eta}$ satisfying 1)-4) and let us show how to obtain $\alpha_{\eta}, \beta_{\eta}$. As $A_{\eta} \subseteq M$ and $M$ is closed under its countable sets we have $A_{\eta} \in M$. Also $f_{A_{\eta}, \gamma} \in M$ as $M$ is closed under countable sets. Hence, by the elementarity there is $\beta_{\eta} \in M \backslash \sup \left(A_{\eta}\right)$ such that $f_{A_{\eta}, \beta_{\eta}}=f_{A_{\eta}, \gamma}$ and $c f\left(\beta_{\eta}\right)=\omega_{1}$. Now $f\left(\left\{\beta_{\eta}, \gamma\right\}\right) \cap M$ is in $M$ again, so using the fact that $\operatorname{cf}\left(\beta_{\eta}\right)=\omega_{1}$ we can find $\alpha_{\eta} \in M$ satisfying $A_{\eta}<\alpha_{\eta}<\beta_{\eta}$ and $\alpha_{\eta} \notin f\left(\left\{\beta_{\eta}, \gamma\right\}\right)$, which completes the construction.

Now define $\gamma_{\eta}<\omega_{1}$ such that for $\xi<\eta<\omega_{1}$ we have

$$
\gamma_{\xi}<\gamma_{\eta} \notin \bigcup\left\{f\left(\left\{\beta_{\xi}, \beta_{\eta}\right\}\right): \xi<\eta\right\} .
$$

Finally define $\mathcal{A}=\left\{\left\{\gamma_{\xi}, \alpha_{\xi}, \beta_{\xi}\right\}: \xi<\omega_{1}\right\}$. Suppose $\xi<\eta$. Note that, as by 4$)$, $\alpha_{\xi} \notin f\left(\left\{\beta_{\xi}, \gamma\right\}\right)$ and by 3$), f\left(\left\{\beta_{\xi}, \gamma\right\}\right)=f\left(\left\{\beta_{\xi}, \beta_{\eta}\right\}\right)$, we have

$$
\alpha_{\xi} \in\left(\beta_{\xi} \cap \beta_{\eta}\right) \backslash f\left(\left\{\beta_{\xi}, \beta_{\eta}\right\}\right) \text {. }
$$

But on the other hand, by the definition of $\gamma_{\eta}$, we have

$$
\gamma_{\eta} \in\left(\beta_{\xi} \cap \beta_{\eta}\right) \backslash f\left(\left\{\beta_{\xi}, \beta_{\eta}\right\}\right),
$$

which shows that the inclusion $\left.{ }^{*}\right)$ of the proposition holds for no $a, b \in \mathcal{A}$. 


\section{REFERENCES}

1. J. E. Baumgartner, Applications of the proper forcing axiom, Handbook of set-theoretic topology, North-Holland, Amsterdam, 1984, pp. 913-959. MR776640 (86g:03084)

2. J. E. Baumgartner and S. Shelah, Remarks on superatomic Boolean algebras, Ann. Pure Appl. Logic 33 (1987), no. 2, 109-129. MR874021 (88d:03100)

3. J. M. Borwein and J. D. Vanderwerff, Banach spaces that admit support sets, Proc. Amer. Math. Soc. 124 (1996), no. 3, 751-755. MR1301010 (96f:46016)

4. R. Deville, G. Godefroy, and V. Zizler, Smoothness and renormings in Banach spaces, Pitman Monographs and Surveys in Pure and Applied Mathematics, vol. 64, Longman Scientific \& Technical, Harlow, 1993.

5. A. Dow, An introduction to applications of elementary submodels to topology, Topology Proc. 13 (1988), no. 1, 17-72. MR.1031969 (91a:54003)

6. P. Hájek, V. Montesinos Santalucía, J. Vanderwerff, and V. Zizler, Biorthogonal systems in Banach spaces, CMS Books in Mathematics/Ouvrages de Mathématiques de la SMC, 26, Springer, New York, 2008.

7. R. Haydon, A counterexample to several questions about scattered compact spaces, Bull. London Math. Soc. 22 (1990), no. 3, 261-268. MR.1041141 (91h:46045)

8. R. Hodel, Cardinal functions. I, Handbook of set-theoretic topology, North-Holland, Amsterdam, 1984, pp. 1-61. MR776620 (86j:54007)

9. T. Jech, Set theory, Springer Monographs in Mathematics, Springer-Verlag, Berlin, 2003, The third millennium edition, revised and expanded. MR.1940513 (2004g:03071)

10. M. Jiménez Sevilla and J.-P. Moreno, Renorming Banach spaces with the Mazur intersection property, J. Funct. Anal. 144 (1997), no. 2, 486-504. MR.1432595 (98a:46024)

11. I. Juhász and L. Soukup, How to force a countably tight, initially $\omega_{1}$-compact and noncompact space?, Topology Appl. 69 (1996), no. 3, 227-250. MR1382294 (97c:54004)

12. I. Juhász and W. Weiss, On thin-tall scattered spaces, Colloq. Math. 40 (1978/79), no. 1, 63-68. MR529798 (82k:54005)

13. W. Just, Two consistency results concerning thin-tall Boolean algebras, Algebra Universalis 20 (1985), no. 2, 135-142. MR806609 (87c:03101)

14. P. Koszmider, Semimorasses and nonreflection at singular cardinals, Ann. Pure Appl. Logic 72 (1995), no. 1, 1-23. MR1320107 (96i:03043)

15. , On strong chains of uncountable functions, Israel J. Math. 118 (2000), 289-315. MR:1776085 (2001g:03091)

16. Universal matrices and strongly unbounded functions, Math. Res. Lett. 9 (2002), no. 4, 549-566. MR 1928875 (2003g:03078)

17. J. C. Martínez, A consistency result on thin-very tall Boolean algebras, Israel J. Math. 123 (2001), 273-284. MR 1835300 (2003c:03090)

18. S. Mazur, Über schwache Konvergenz in den Raumen $L^{p}$, Studia Math. 4 (1933), 129-133.

19. I. Namioka and R. R. Phelps, Banach spaces which are Asplund spaces, Duke Math. J. 42 (1975), no. 4, 735-750. MR0390721 (52:11544)

20. S. Negrepontis, Banach spaces and topology, Handbook of set-theoretic topology, NorthHolland, Amsterdam, 1984, pp. 1045-1142. MR776642 (86i:46018)

21. A. J. Ostaszewski, A countably compact, first-countable, hereditarily separable regular space which is not completely regular, Bull. Acad. Polon. Sci. Sér. Sci. Math. Astronom. Phys. 23 (1975), no. 4, 431-435. MR0377815 (51:13984)

22. M. Rabus, An $\omega_{2}$-minimal Boolean algebra, Trans. Amer. Math. Soc. 348 (1996), no. 8, 3235-3244. MR1357881 (96j:03070)

23. M. Rajagopalan, A chain compact space which is not strongly scattered, Israel J. Math. 23 (1976), no. 2, 117-125. MR0402701 (53:6517)

24. J. Roitman, Introduction to modern set theory, Pure and Applied Mathematics (New York), John Wiley \& Sons Inc., New York, 1990, A Wiley-Interscience Publication. MR.1028781 (91h:03003)

25. B. Ė. Shapirovskiǔ, Cardinal invariants in compacta, Seminar on General Topology, Moskov. Gos. Univ., Moscow, 1981, pp. 162-187. (Russian) MR656957 (83f:54024)

26. S. Shelah, Proper forcing, Lecture Notes in Mathematics, vol. 940, Springer-Verlag, Berlin, 1982. MR675955 (84h:03002) 
27. S. Todorcevic, A note on the proper forcing axiom, Axiomatic set theory (Boulder, Colo., 1983), Contemp. Math., vol. 31, Amer. Math. Soc., Providence, RI, 1984, pp. 209-218. MR.763902 (86f:03089)

28. MR792822 (87a:03084)

29. Biorthogonal systems and quotient spaces via Baire category methods, Math. Ann. 335 (2006), no. 3, 687-715. MR2221128 (2007d:46016)

30. D. Velleman, Simplified morasses, J. Symbolic Logic 49 (1984), no. 1, 257-271. MR736620 (85i:03162)

31. W. S. Zwicker, $P_{k} \lambda$ combinatorics. I. Stationary coding sets rationalize the club filter, Axiomatic set theory (Boulder, Colo., 1983), Contemp. Math., vol. 31, Amer. Math. Soc., Providence, RI, 1984, pp. 243-259. MR763904 (86e:03046)

Institute of Mathematics, Statistics and Scientific Computing, Universidade Estadual de Campinas, Caixa Postal 6065, 13083-970, Campinas, SP, Brazil

E-mail address: christina.brech@gmail.com

Current address: Instituto de Matemática e Estatística, Universidade de São Paulo, Caixa Postal 66281, 05314-970, São Paulo, SP, Brazil

Instytut Matematyki, Politechnika Łódzka, Ul. Wólczańska 215; 90-924 Łódź, PolAND

E-mail address: pkoszmider.politechnika@gmail.com 